Published in final edited form as:

Evid Based Med. 2016 April ; 21(2): 80. doi:10.1136/ebmed-2015-110373.

\title{
Early exposure to dogs and farm animals reduces risk of childhood asthma
}

\author{
Iny Jhun ${ }^{1}$ and Wanda Phipatanakul ${ }^{1,2}$ \\ ${ }^{1}$ Harvard Medical School, Boston, Massachusetts, USA \\ 2Division of Allergy and Immunology, Department of Pediatrics, Boston's Children's Hospital, \\ Boston, Massachusetts, USA
}

Context

Asthma is one of the most common chronic diseases of childhood. The risk of developing asthma is associated with environmental exposures, including tobacco smoke, air pollution, dust mites, endotoxin and farm animals. ${ }^{1}$ A lower prevalence of asthma among children in farm environments supports the hygiene hypothesis on the role of childhood exposure to microbes in developing a more favourable immune system. The effect of exposures to domestic dogs has been less clear. Fall et al undertook a nationwide study to investigate the association between early exposures to dogs as well as farm environment and risk of childhood asthma.

\section{Methods}

The study population included all children born in Sweden between 2001 and 2010. The study population was divided into two subcohorts: school-aged and preschool-aged children. Dog exposure was determined by a parent registering as a dog owner during the child's first year of life. Farm animal exposure was defined by parents being identified as animal producers and related workers during the child's first year of life. The association between exposure to dogs and farm animals during the first year of life and asthma was assessed as the OR for a current diagnosis of asthma at age 6 years for school-aged children and as the HR for incident asthma at ages 1-5 years for preschool-aged children.

\section{Findings}

There were 376638 preschool-aged and 276298 school-aged children included in the analyses. Dog exposure during the first year of life was associated with a decreased risk of asthma in school-aged children (OR, 0.87; 95\% CI 0.81 to 0.93 ) and in preschool-aged children 3 years or older (HR, $0.90 ; 95 \%$ CI 0.83 to 0.99 ) but not in children younger than 3 years. Farm animal exposure was associated with a reduced risk of asthma in school-aged

Correspondence to: Wanda Phipatanakul, Division of Allergy and Immunology, Department of Pediatrics, Boston's Children's Hospital, 300 Longwood Avenue, Boston, MA 02115, USA; wanda.phipatanakul@ childrens.harvard.edu.

Competing interests None declared.

Provenance and peer review Commissioned; internally peer reviewed. 
children and preschool-aged children (OR, 0.48; 95\% CI 0.31 to 0.76 , and HR, 0.69; $95 \%$ CI 0.56 to 0.84 ), respectively.

\section{Commentary}

Household pet ownership has received considerable attention in its potential role in asthma development, particularly as asthma incidence continues to rise in countries where pet ownership is common. The hygiene hypothesis has been supported by many studies demonstrating the protective effect of farm animal exposure against childhood asthma. ${ }^{2}$ However, studies on dog exposure have been inconclusive and limited by power and availability of longitudinal data. ${ }^{3}$

Fall et al found a reduced risk of childhood asthma in association with early exposure to dog as well as farm animals, supporting the hygiene hypothesis. Authors demonstrated robustness of the results after stratifying by parental asthma.

Consistent with prior studies, the authors demonstrated a significant reduction in asthma risk with farm animal exposure for school-aged and preschool-aged children. Dog exposure in the first year of life reduced the risk of childhood asthma for school-aged children, but not for children under 3. Furthermore, there was an increased risk of pneumonia and respiratory tract infections in association with dog exposure in preschool-aged children. In addition, differential effects of dog exposure on atopic versus non-atopic asthma have been reported, ${ }^{4}$ but it was not possible to make this distinction in this study.

Lastly, the results of this study may not be generalisable to low-income countries. Prior studies have found reduced risk of childhood asthma with farm animal exposure in highincome countries, but some evidence of an increased risk in low-income countries. ${ }^{15}$

Further studies are needed to understand what specifically about dog exposure supports this protective association and whether this is a marker for other protective exposures (ie, allergens, bacterial/viral microbes, endotoxin, etc).

\section{Implications for practice}

Specific recommendations on pet ownership with regards to childhood asthma risk cannot be made from this study, as different studies have shown conflicting results. However, the results of this study are provocative in support of early dog exposure as protective in asthma.

\section{Acknowledgments}

Funding NIH K24 AI 106822.

\section{References}

1. Beasley R, Semprini A, Mitchell EA. Risk factors for asthma: is prevention possible? Lancet. 2015; 386:1075-85. [PubMed: 26382999]

2. von Mutius E, Vercelli D. Farm living: effects on childhood asthma and allergy. Nat Rev Immunol. 2010; 10:861-8. [PubMed: 21060319] 
3. Lødrup Carlsen KC, Roll S, Carlsen KH, et al. Does pet ownership in infancy lead to asthma or allergy at school age? Pooled analysis of individual participant data from 11 European birth cohorts. PLoS ONE. 2012; 7:e43214. [PubMed: 22952649]

4. Collin SM, Granell R, Westgarth C, et al. Pet ownership is associated with increased risk of nonatopic asthma and reduced risk of atopy in childhood: findings from a UK birth cohort. Clin Exp Allergy. 2015; 45:200-10. [PubMed: 25077415]

5. Genuneit J. Exposure to farming environments in childhood and asthma and wheeze in rural populations: a systematic review with meta-analysis. Pediatr Allergy Immunol. 2012; 23:509-18. [PubMed: 22625206] 